\title{
Stratified Prognostic Value of Pathological Response to Preoperative Treatment in yp II/III Rectal Cancer
}

\author{
Yanpeng Yang ${ }^{\dagger}$, Hao Xu ${ }^{\dagger}$, Guowei Chen ${ }^{*}$ and Yisheng Pan * \\ Department of Gastroenterology, Peking University First Hospital, Beijing, China
}

OPEN ACCESS

Edited by:

Silvia R. Rogatto,

University of Southern Denmark,

Denmark

Reviewed by:

Thomas Samuel Ram,

Christian Medical College \& Hospital,

Marco Massani,

ULSS2 Marca Trevigiana, Italy

*Correspondence:

Guowei Chen

1911110294@pku.edu.cn

Yisheng Pan

yishengpan@bjmu.edu.cn

${ }^{\dagger}$ These authors have contributed equally to this work and share first authorship

Specialty section:

This article was submitted to Gastrointestinal Cancers:

Colorectal Cancer,

a section of the journa

Frontiers in Oncology

Received: 14 October 2021 Accepted: 29 November 2021 Published: 16 December 2021

Citation:

Yang $Y, X u H$, Chen $G$ and Pan Y (2021) Stratified Prognostic Value of Pathological Response to Preoperative Treatment in yp II/III Rectal Cancer.

Front. Oncol. 11:795137. doi: 10.3389/fonc.2021.795137
Aim: Accumulated studies have verified that tumor regression is associated with the prognosis of rectal cancer. However, stratified analysis within a certain stage is still unknown. The purpose of our study was to assess the impact of pathologic response on the survival of stagell and III rectal cancer patients after neoadjuvant chemoradiotherapy (nCRT).

Methods: Clinicopathologic characteristics and tumor regression scores (TRS) were assessed in 236 rectal cancer patients who treated with nCRT followed by surgery. Survival analysis was performed using Cox proportional hazards models.

Results: Among these patients, the stage of 88 patients was ypll, and 91 patients were with the stage of yplll. The median follow-up time was 59.8 months. TRS was not an independent prognostic factor in ypll patients while it was significantly associated with the prognosis of yplll patients (5-year survival rate $67.2 \%$ vs. $42.5 \%, P<0.001$ ). Furthermore, yplll patients with the response to nCRT had similar survival to that of ypll patients (5-year survival rate $67.2 \%$ vs. $70.5 \%, P=0.56$ ). For yplll patients, multivariable analysis showed that well differentiation, negative surgical margin, and the administration of adjuvant chemotherapy were associated with better survival. The surgical margin and differentiation were prognostic factors for ypll patients.

Conclusions: yplll rectal cancer patients with poor response to preoperative treatment are at high risk of worse oncological outcomes.

Keywords: rectal cancer, survival, neoadjuvant (chemo)radiotherapy, tumor regression grade (TRG), prognosis

\section{INTRODUCTION}

Neoadjuvant chemoradiotherapy (nCRT) was recommended in the 1990s to treat the patients with locally advanced rectal cancer. Compared to the conventional surgery combined with postoperative therapy, nCRT has been proved more effective in local control, downstaging, and sphincter sparing $(1,2)$. The pathologic stage of the resection specimen (yp) is the strongest prognostic factor for patients who underwent neoadjuvant therapy. The ypI patients usually possess long-term survival and local control, while $30 \%$ of ypII and III rectal cancer patients will suffer from relapse (3). 
Therefore, physicians need to find a prognostic factor that can precisely predict the survival of high-risk individuals. It will be benefit to decide the adjuvant regimen and appropriate surveillance intervals. Until now, the most accurate method for evaluating the response is the histological change of the resected specimen. These changes include cytologic and stromal alterations, such as cytoplasmic vacuolation and mucus lakes at the site of previous tumors $(4,5)$. Tumor regression can range from no evidence of therapeutic effect to complete response with no residual cancer cells. To conveniently describe the response to nCRT, the tumor regression score (TRS) was introduced to evaluate the degree of remission. According to previous researches, patients with pathologic complete remission ( $\mathrm{pCR}$ ) generally have superior long-term survival and at low risk of relapse. Patients with moderate, minimal, or no response have progressively worse outcomes $(4,6)$.

Accumulated trials have verified that well pathological responses, such as pCR and ypI, are associated with better outcomes. However, the survival of ypII and III rectal cancer patients are variable. They maybe experience recurrence at a rate of $20-30 \%(7-11)$. Thus, only depending on yp stage is not enough to identify the high-risk population. Many published studies have confirmed the prognostic value of the response to neoadjuvant treatment (4), but a stratified analysis within a certain stage is still unknown. We hypothesized that well response to nCRT would be associated with improved survival among specific patients. Therefore, the purpose of our study was to assess the impact of nCRT response on the survival of ypII and ypIII rectal cancer patients, which could help to identify highrisk rectal cancers.

\section{METHODS}

\section{Patients and Data Sources}

A total of 256 pathologically confirmed rectal cancer patients who were treated with nCRT followed by surgery in Peking University First Hospital between 2008 and 2019 were collected in our study. The medical records and surveillance data were obtained prospectively. This had been approved by the institutional review board. Exclusion criteria included incomplete nCRT or surgery, stage IV disease, history of other cancers, and insufficient data.

\section{Treatment}

Rectal cancer with clinical stage of T3-4 or N+ was defined as locally advanced rectal cancer. Treatments for locally advanced rectal cancer patients were decided by multidisciplinary team (MDT) discussions. The team consisted of professional oncologists, surgeons, radiologists, and pathologists. The patients involved in this research received concurrent chemotherapy with radiation, usually oral capecitabine or intravenous 5-FU and a long course of 50.4 Gy radiation in 25 fractions, followed by surgery with curative intent. Surgeries complied with the principle of total mesorectal excision (TME). And the interval between the last treatment and surgery was about 8 weeks for all the patients. All patients were encouraged to receive adjuvant chemotherapy after surgery. The regimens of adjuvant chemotherapy were CapeOX or FOLFOX. And up to 6 months of perioperative chemotherapy was recommended.

Response to nCRT was evaluated by experienced pathologists without knowing the outcomes of the patients. The system used to grade the tumor response was recommended by the AJCC Cancer Staging Manual (8th Edition) and the College of American Pathologists (CAP) guidelines: tumor regression score 0 (TRS 0) (complete response), no remaining viable cancer cells; TRS 1 (moderate response), only small clusters or single cancer cells remaining; TRS 2 (minimal response), residual cancer remaining, but with predominant fibrosis; TRS 3 (poor response), minimal or no tumor kill, extensive residual cancer.

\section{Statistical Analysis}

Clinicopathologic characteristics and oncologic outcomes of the populations were collected and analyzed. The association between these factors and TRS was assessed by the chi-square, Fisher exact, and Mann-Whitney $U$ tests, as appropriate. Survival was estimated using the log-rank test. Variables were selected into the multivariable model depend on statistical significance $(P<0.2)$, and the stepwise Cox regression model was used. All analyses were carried out with IBM SPSS version 27.0. Statistically significant was considered when a two-tailed $P$ value less than 0.05 .

\section{RESULTS}

\section{Patient Characteristics}

A total of 256 rectal cancer patients were involved in our research, and the prognosis and characteristics were further analyzed. The median follow-up time was 59.8 months, and the 3 - and 5-year overall survivals of the entire population were $78.5 \%$ and $69.3 \%$, respectively. After nCRT, 31 patients (12.1\%) achieved pCR (TRS 0), whereas 58(22.7\%), 96 (37.5\%), and 71 (27.7\%) patients had the TRS of 1,2 , and 3 , respectively. The pathological differentiation in most patients was moderate and poor $(69.8 \%)$. After surgery, $40.7 \%$ of patients received adjuvant chemotherapy.

\section{Characteristics and Survival Analysis of ypll}

Stratified by tumor regression scores, clinical and pathological characteristics of ypII rectal cancer patients were summarized in Table 1. Eighty-eight patients were staged ypII, and most of the patients were at ypT3 stage (78.4\%). In addition to this, only $37.5 \%$ of ypII patients received adjuvant chemotherapy. And predictors of pathologic response were gender $(P=0.03)$ and tumor size $(P=0.03)$.

The 5-year survival rate of ypII patients was $70.5 \%$, with a median survival of 101.4 months. Variables associated with survival after surgery in ypII patients were illustrated in Table 2. TRS 1 and 2 were grouped and compared with TRS 3. In the univariable analysis, we found that the TRS was associated 
TABLE 1 | Clinicopathologic characteristics of ypll patients.

\begin{tabular}{|c|c|c|c|c|}
\hline \multirow[t]{2}{*}{ Variables } & \multicolumn{3}{|c|}{ Tumor regression score } & \multirow[t]{2}{*}{$P$ value } \\
\hline & 1 & 2 & 3 & \\
\hline Age (88) & & & & 0.19 \\
\hline$\geq 65$ (34) & 13 & 11 & 10 & \\
\hline$<65(54)$ & 14 & 28 & 12 & \\
\hline Gender (88) & & & & 0.03 \\
\hline Male (59) & 23 & 21 & 15 & \\
\hline Female (29) & 4 & 18 & 7 & \\
\hline BMI, kg/m² (88) & & & & 0.38 \\
\hline$<24$ (53) & 15 & 22 & 16 & \\
\hline$\geq 24(35)$ & 12 & 17 & 6 & \\
\hline Clinical T stage (88) & & & & 0.98 \\
\hline $\mathrm{T} 1(6)$ & 1 & 3 & 2 & \\
\hline T2 (15) & 5 & 6 & 4 & \\
\hline T3 (38) & 13 & 16 & 9 & \\
\hline T4 (29) & 8 & 14 & 7 & \\
\hline Clinical N stage (88) & & & & 0.20 \\
\hline No (22) & 4 & 12 & 6 & \\
\hline N1 (56) & 17 & 24 & 15 & \\
\hline N2 (10) & 6 & 3 & 1 & \\
\hline Clinical stage (88) & & & & 0.33 \\
\hline II (22) & 4 & 12 & 6 & \\
\hline III (66) & 23 & 27 & 16 & \\
\hline Procedure (88) & & & & 0.45 \\
\hline LAR (56) & 20 & 23 & 13 & \\
\hline APR (21) & 3 & 12 & 6 & \\
\hline Combined resection (11) & 4 & 4 & 3 & \\
\hline Distance from anal verge, $\mathrm{cm}(87)$ & & & & 0.06 \\
\hline$\geq 5(62)$ & 24 & 25 & 14 & \\
\hline$<5(25)$ & 3 & 14 & 8 & \\
\hline Tumor size, cm & & & & 0.03 \\
\hline$\geq 3.5(27)$ & 3 & 16 & 8 & \\
\hline$<3.5(61)$ & 24 & 23 & 14 & \\
\hline Pathological T stage (88) & & & & 0.69 \\
\hline T3 (69) & 21 & 32 & 16 & \\
\hline T4 (19) & 6 & 7 & 6 & \\
\hline Histological differentiation (83) & & & & 0.90 \\
\hline Well (4) & 1 & 3 & 0 & \\
\hline Moderate (64) & 18 & 29 & 17 & \\
\hline Poor (15) & 5 & 6 & 4 & \\
\hline Positive lymphovascular invasion & 1 & 3 & 2 & 0.77 \\
\hline Positive surgical margin & 1 & 4 & 4 & 0.28 \\
\hline
\end{tabular}

with survival $(P=0.03)$. However, there was no statistical difference between them with multivariate analysis (Figure 1A, $P=0.15)$. In univariable and multivariable analysis, only the histologic differentiation (HR 4.17, 95\% CI 0.71-6.25, $P=0.024$ ) and surgical margin (HR 2.78, 95\% CI $0.35-5.26, P<0.001$ ) remained difference significantly.

\section{Characteristics and Survival Analysis of yplll}

Stratified by tumor regression scores, clinical and pathological characteristics of ypIII rectal cancer were summarized in Table 3. Ninety-one patients were staged ypIII. Four patients achieved a local pathologically complete response, whereas the lymph nodes harvested were positive. The predictors of pathologic response were the clinical nodal status $(P=0.03)$, stage $(P=0.047)$, and the pathologic T stage $(P<0.001)$.

The 5-year survival rate of ypIII patients was $63.6 \%$, with a median survival of 79.8 months. Variables associated with survival after surgery in ypIII patients were illustrated in Table 4 . In the same way, TRS 0,1 , and 2 were grouped and compared with TRS 3 for analysis. TRS was associated with survival in the univariable analysis $(P<0.001)$. There was also a significant difference between them in multivariate analysis (HR 2.63, 95\% CI 1.12-5.88, $P<0.001$, Figure 1B). Besides, younger age, well histological differentiation, low anterior resection, negative surgical margin, and the completion of adjuvant chemotherapy were associated with better survival in univariable analysis. Negative surgical margin, well differentiation, and the presence of adjuvant chemotherapy remained statistically significant in multivariable analysis.

In order to compare the survival of ypII and ypIII rectal cancer patients, the survival curves were calculated together, which were stratified by response to nCRT (TRS 0-2) and no response. The overall survival of ypIII patients with response was not significantly different from ypII disease (Figure 1C, $P=0.56)$. 
TABLE 2 | Analysis of factors associated with overall survival for ypll patients.

\begin{tabular}{|c|c|c|c|c|c|c|}
\hline \multirow[t]{2}{*}{ Variables } & \multirow[t]{2}{*}{ Median survival (m) } & \multirow[t]{2}{*}{ 5-year Survival (\%) } & \multirow[t]{2}{*}{ Log-rank test } & \multicolumn{3}{|c|}{ Cox multivariate test } \\
\hline & & & & HR & $95 \% \mathrm{Cl}$ & $P$ \\
\hline Age & & & 0.59 & & & \\
\hline$\geq 65$ & 80.2 & 69.0 & & & & \\
\hline$<65$ & 101.4 & 71.2 & & & & \\
\hline Gender & & & 0.72 & & & \\
\hline Male & 92.1 & 79.6 & & & & \\
\hline Female & 85.6 & 75.4 & & & & \\
\hline BMI, kg/m² & & & 0.61 & & & \\
\hline$<24$ & 97.4 & 73.0 & & & & \\
\hline$\geq 24$ & 74.5 & 68.6 & & & & \\
\hline Clinical stage & & & 0.06 & & & \\
\hline$\|$ & 98.7 & 82.0 & & & & \\
\hline III & 83.6 & 70.5 & & & & \\
\hline Procedure & & & 0.04 & & & \\
\hline LAR & 103.5 & 81.6 & & Ref & & \\
\hline APR & 90.3 & 65.4 & & 1.20 & $0.82-3.67$ & 0.21 \\
\hline Combined resection & 77.5 & 43.8 & & 1.32 & $0.45-1.79$ & 0.056 \\
\hline Distance from anal verge & & & 0.91 & & & \\
\hline$\geq 5 \mathrm{~cm}$ & 101.3 & 72.7 & & & & \\
\hline$<5 \mathrm{~cm}$ & 87.5 & 69.1 & & & & \\
\hline Tumor size, cm & & & 0.68 & & & \\
\hline$\geq 3.5$ & 84.4 & 69.0 & & & & \\
\hline$<3.5$ & 104.1 & 71.7 & & & & \\
\hline Pathological T stage & & & 0.34 & & & \\
\hline T3 & 101.0 & 70.5 & & & & \\
\hline $\mathrm{T} 4$ & 82.8 & 70.0 & & & & \\
\hline Histological differentiation & & & 0.006 & & & \\
\hline Well/moderate & 111.7 & 82.7 & & Ref & & \\
\hline Poor & 71 & 70.5 & & 4.17 & $0.71-6.25$ & 0.024 \\
\hline Lymphovascular invasion & & & 0.64 & & & \\
\hline Negative & 100.7 & 83.3 & & & & \\
\hline Positive & 49.2 & 69.5 & & & & \\
\hline Surgical Margin & & & $<0.001$ & & & \\
\hline Negative & 98.3 & 76.5 & & Ref & & \\
\hline Positive & NR & 25.0 & & 2.78 & $0.35-5.26$ & $<0.001$ \\
\hline Adjuvant chemotherapy & & & 0.72 & & & \\
\hline No & 102.7 & 69.6 & & & & \\
\hline Yes & 66.6 & 71.9 & & & & \\
\hline Tumor regression score & & & 0.03 & & & \\
\hline $0-2$ & 103.8 & 72.3 & & Ref & & \\
\hline 3 & 80.6 & 65.2 & & 1.16 & $0.83-2.77$ & 0.15 \\
\hline
\end{tabular}

$N R$, not reached.

\section{DISCUSSION}

Based on previous studies, the tumor regression score after neoadjuvant chemoradiation was a significant independent prognostic factor for rectal cancer patients. Patients with no response to nCRT had the 5-year survival rate of $27 \%$ compared to $72 \%$ for patients with response (12). Similarly, in the CAO/ ARO/AIO-94 trial, pCR patients had a 10-year disease-free survival of $89.5 \%$, while those with poor regression had a corresponding incidence of $63 \%$ (13). The nCRT response had other predictive values in addition to predict the survival. In the EORTC 22921 trial, a subgroup analysis showed that ypT0-2 patients were more likely to benefit from adjuvant chemotherapy than ypT3-4 patients (8). Although the predictive value of the tumor regression score has been reported, a classification analysis of ypTNM stage has not been mentioned. Our current study analyzed the prognostic value of the tumor regression score classified by pathologic stage for the first time.

To investigate the impact of the tumor regression score on the classification stage, the patients were divided into two groups in each stage: response (TRS 0-2) and no response (TRS 3). The independent prognostic factor for ypII patients was histological grade. For patients at stage III who received nCRT, response to nCRT, well histological differentiation, negative surgical margin, and completion of adjuvant chemotherapy were all independently associated with improved survival. The differentiation and surgical margin but not the response to nCRT were consistent predictors of survival in both ypII and III patients. We found that ypIII patients with the response to nCRT had similar survival to that of ypII patients. However, it was difficult to distinguish the survival between response and no response among ypII patients. 
A

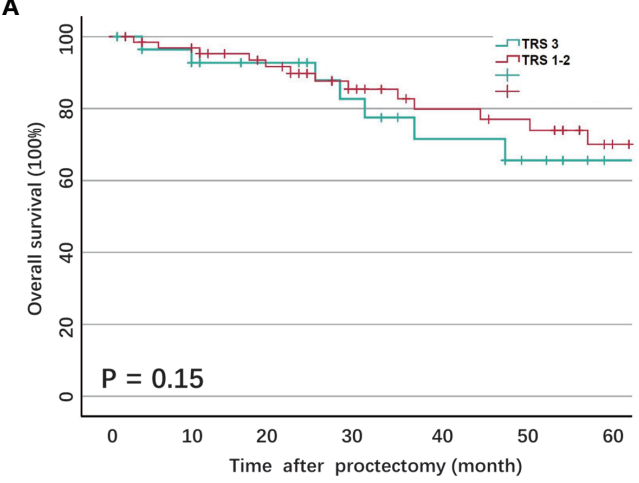

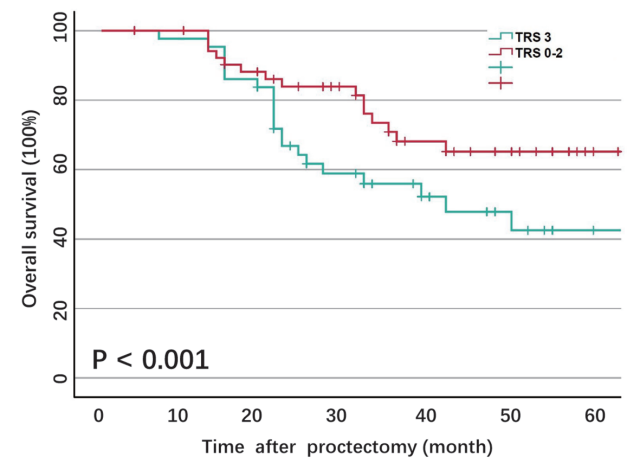

C

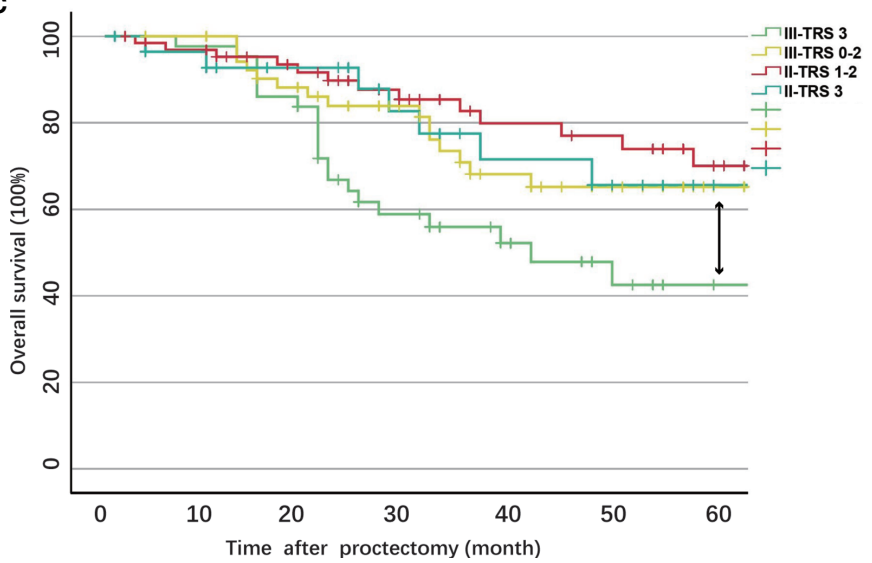

FIGURE 1 | (A) Overall survival of ypll patients stratified by tumor regression score; (B) Overall survival of ypll patients stratified by tumor regression score; (C) Overall survival of yplll patients compared with ypll patients stratified by tumor regression score.

It is known that surgical resection with negative margins in rectal cancer is critical because treatment modalities, such as chemotherapy and radiation, cannot compensate for a positive margin. The relationship between resection margin and local recurrence and survival has been reported by many studies. Our results substantiated the surgical margin as a prognostic factor of rectal cancer with nCRT. For histological tumor differentiation, well or moderate differentiation refer to cancer cells with a low invasive property. Poor differentiation is related to more aggressive cancer cells than the former. It is obvious that patients with poorer differentiated cancers suffer from worse oncologic outcomes in most cases.

It is necessary to investigate the reason for distinct outcomes of ypIII patients. According to the definition, ypIII tumors have cancer cells extending from the primary tumor location to the regional lymph nodes. Compared to ypII disease, ypIII tumors prefer to disseminate to other areas and are less likely to be solved by surgery alone. nCRT response could help us to discover the biology of tumors, which may act as an indicator of susceptibility to adjuvant chemotherapy. Both chemotherapy and neoadjuvant treatment could diminish recurrence by eradicating cancer cell that transferred to the lymphatic and blood vessels of patients with response to nCRT. Because of the resistance to cytotoxic agents, patients with no response had great possibility of recurrence. It was the inherent characteristics of the non-response ypIII patients that determined their prognosis. Whereas ypII tumors with disease located in the primary site and could be cured by surgery with a great possibility. This mostly clarified the mechanism that ypIII rectal cancer patients with response to nCRT had similar survival to ypII patients.

There were also many factors that could predict the degree of response. For ypII patients, there were statistical differences in gender and tumor size. Combined with clinical practice, we were unable to confirm whether the gender of patients was a prognostic factor of the response score, and the number of female patients was obviously less than males in our study. As for tumor size, it was evident that smaller tumors were more likely to show regression than larger tumors when treated by the same regimen. For ypIII patients, the predictive factors of TRS were clinical nodal status, stage, and the pathologic $\mathrm{T}$ stage. We were skeptical of this result because $65.9 \%$ of ypIII patients with T3 stage, and statistical bias could not be ignored. And the clinical stage showed a modest significance, which was unconvincing. Furthermore, we did not find a concurrent predictor of TRS in ypII and III diseases. Previous investigations have reported that TRS was associated with the interval between operation and nCRT completion, and patients 
TABLE 3 | Clinicopathologic characteristics of yplll patients.

\begin{tabular}{|c|c|c|c|c|c|}
\hline \multirow[t]{2}{*}{ Variables } & \multicolumn{4}{|c|}{ Tumor regression score } & \multirow[t]{2}{*}{$P$ value } \\
\hline & 0 & 1 & 2 & 3 & \\
\hline Age (91) & & & & & 0.90 \\
\hline$\geq 65$ (34) & 1 & $9 c$ & 17 & 7 & \\
\hline$<65(54)$ & 3 & 15 & 25 & 14 & \\
\hline Gender (91) & & & & & 0.40 \\
\hline Male (55) & 2 & 18 & 23 & 12 & \\
\hline Female (36) & 2 & 6 & 19 & 9 & \\
\hline BMI, kg/m² (91) & & & & & 0.94 \\
\hline$<24(59)$ & 3 & 16 & 26 & 14 & \\
\hline$\geq 24$ (32) & 1 & 8 & 16 & 7 & \\
\hline Clinical T stage (91) & & & & & 0.38 \\
\hline $\mathrm{T} 1$ & 0 & 0 & 2 & 1 & \\
\hline $\mathrm{T} 2$ & 2 & 4 & 5 & 6 & \\
\hline T3 & 2 & 11 & 26 & 10 & \\
\hline T4 & 0 & 9 & 9 & 4 & \\
\hline Clinical N stage (89) & & & & & 0.03 \\
\hline NO & 3 & 3 & 8 & 5 & \\
\hline N1 & 1 & 11 & 26 & 14 & \\
\hline $\mathrm{N} 2$ & 0 & 9 & 7 & 2 & \\
\hline Clinical stage (89) & & & & & 0.047 \\
\hline II (19) & 3 & 3 & 8 & 5 & \\
\hline III (70) & 1 & 20 & 33 & 16 & \\
\hline Procedure (91) & & & & & 0.90 \\
\hline LAR (60) & 3 & 15 & 29 & 13 & \\
\hline APR (27) & 1 & 8 & 12 & 6 & \\
\hline Combined resection (4) & 0 & 1 & 1 & 2 & \\
\hline Distance from anal verge, $\mathrm{cm}(91)$ & & & & & 0.91 \\
\hline$\geq 5(61)$ & 3 & 17 & 28 & 13 & \\
\hline$<5(30)$ & 1 & 7 & 14 & 8 & \\
\hline Tumor size, cm (91) & & & & & 0.71 \\
\hline$\geq 3.5(27)$ & 1 & 6 & 15 & 5 & \\
\hline$<3.5(64)$ & 3 & 18 & 27 & 16 & \\
\hline Pathological T stage (91) & & & & & $<0.001$ \\
\hline $\mathrm{TO}(4)$ & 4 & 0 & 0 & 0 & \\
\hline $\mathrm{T} 1(2)$ & 0 & 1 & 1 & 0 & \\
\hline $\mathrm{T} 2(19)$ & 0 & 6 & 10 & 3 & \\
\hline T3 (60) & 0 & 14 & 29 & 17 & \\
\hline $\mathrm{T} 4(6)$ & 0 & 3 & 2 & 1 & \\
\hline Pathological N stage & & & & & 0.94 \\
\hline N1 (59) & 3 & 16 & 26 & 14 & \\
\hline N2 (32) & 1 & 8 & 16 & 7 & \\
\hline Histological differentiation (89) & & & & & 0.18 \\
\hline Well (6) & 1 & 2 & 3 & 0 & \\
\hline Moderate (56) & 2 & 16 & 27 & 11 & \\
\hline Poor (27) & 0 & 6 & 11 & 10 & \\
\hline Positive Lymphovascular invasion & 0 & 1 & 4 & 3 & 0.63 \\
\hline Positive surgical margin & 0 & 0 & 2 & 3 & 0.20 \\
\hline
\end{tabular}

with longer intervals were more likely to have lower tumor regression scores $(14,15)$. Most of the patients in our study underwent surgeries with interval about 8 weeks after the last time of chemoradiation. There was no difference of the interval between surgery and the last preoperative treatment of the patients, so we did not take it into consideration.

Regardless of the prognostic value of the TRS, its clinical implications are also important. In view of the poor response of some rectal cancer patients, they may not benefit from adjuvant cytotoxic therapy. However, with no alternative options, they could probably receive more intensive adjuvant therapies or participate in novel therapeutic trials. The panel of National Comprehensive Cancer Network (NCCN) believes that patients with tumor downstaging and complete response after nCRT should be strongly considered for adjuvant chemotherapy ( 8 , 16). In addition to the choice of adjuvant therapy, the fact that long-term outcomes of the non-responders vary from patients with a response suggests that more rigorous surveillance is necessary for this population.

There were also some limitations of our study. Because this was a retrospective study, there was potential bias introduced by the loss of follow-up as well as from the variable collection of data. As part of the cancer database, our data were collected prospectively, which might help reduce the data bias to a certain extent. In addition, adjuvant chemotherapy regimens were not administrated based on the single treatment protocol. Some 
TABLE 4 | Analysis of factors associated with overall survival for yplll patients.

\begin{tabular}{|c|c|c|c|c|c|c|}
\hline \multirow[t]{2}{*}{ Variables } & \multirow[t]{2}{*}{ Median Survival (m) } & \multirow[t]{2}{*}{ 5-year survival (\%) } & \multirow[t]{2}{*}{ Log-rank test } & \multicolumn{3}{|c|}{ Cox multivariate test } \\
\hline & & & & HR & $95 \% \mathrm{Cl}$ & $P$ \\
\hline Age & & & 0.04 & & & \\
\hline$\geq 65$ & 69.3 & 64.0 & & Ref & & \\
\hline$<65$ & 75.7 & 70.1 & & 0.96 & $0.23-2.89$ & 0.56 \\
\hline Gender & & & 0.93 & & & \\
\hline Male & 79.3 & 62.3 & & & & \\
\hline Female & 61.0 & 64.7 & & & & \\
\hline Clinical stage & & & 0.12 & & & \\
\hline$\|$ & 78.8 & 76.3 & & & & \\
\hline III & 69.3 & 62.5 & & & & \\
\hline Procedure & & & 0.013 & & & \\
\hline LAR & 82.1 & 75.6 & & Ref & & \\
\hline APR & 64.5 & 63.2 & & 1.05 & $0.52-1.33$ & 0.06 \\
\hline Combined resection & NA & - & & & & \\
\hline Distance from anal verge & & & 0.80 & & & \\
\hline$\geq 5 \mathrm{~cm}$ & 79.0 & 61.7 & & & & \\
\hline$<5 \mathrm{~cm}$ & 58.2 & 65.0 & & & & \\
\hline Tumor size, cm & & & 0.51 & & & \\
\hline$\geq 3.5$ & 73.5 & 65.4 & & & & \\
\hline$<3.5$ & 68.3 & 61.2 & & & & \\
\hline Pathological T stage & & & 0.32 & & & \\
\hline TO & NA & & & & & \\
\hline $\mathrm{T} 1$ & NA & & & & & \\
\hline $\mathrm{T} 2$ & 78.5 & 62.5 & & & & \\
\hline Т3 & 66.3 & 52.7 & & & & \\
\hline $\mathrm{T} 4$ & $N R$ & & & & & \\
\hline Pathological N stage & & & 0.06 & & & \\
\hline N1 & 79.4 & 81.8 & & Ref & & \\
\hline N2 & 64.8 & 36.8 & & 1.26 & $0.33-2.89$ & 0.14 \\
\hline Histological differentiation & & & 0.003 & & & \\
\hline Well/moderate & 68.2 & 71.4 & & Ref & & \\
\hline Poor & 53.4 & 60.6 & & 1.79 & $1.09-2.94$ & 0.02 \\
\hline Lymphovascular invasion & & & 0.64 & & & \\
\hline Negative & 81.2 & 80.1 & & & & \\
\hline Positive & 42.8 & 56.3 & & & & \\
\hline \multicolumn{7}{|l|}{ Surgical Margin } \\
\hline Negative & 79.6 & 78.3 & $<0.001$ & Ref & & \\
\hline Positive & NA & 0 & & 3.57 & $0.56-6.25$ & 0.016 \\
\hline Adjuvant chemotherapy & & & $<0.001$ & & & \\
\hline No & 63.7 & 43.5 & & Ref & & \\
\hline Yes & 82.6 & 85.4 & & 0.35 & $0.13-0.95$ & 0.007 \\
\hline Tumor regression score & & & $<0.001$ & & & \\
\hline $0-2$ & 78.6 & 67.2 & & Ref & & \\
\hline 3 & 56.0 & 42.5 & & 2.63 & $1.12-5.88$ & $<0.001$ \\
\hline
\end{tabular}

NA, not applicable; NR, not reached.

patients received CapeOX, whereas others received FOLFOX. The total pCR rate in our study was $12.1 \%$, which was lower than the reported rates of $16-24 \%$ (16). This might be attributed to the dissimilarity of regimens and the generally late stage of rectal cancer patients in China. And this study reflects the outcomes of a specific population in China, and extending the results to other populations should be prudent.

\section{CONCLUSION}

ypIII rectal cancer patients with poor response to nCRT are at high risk of worse oncological outcomes. More intensive adjuvant chemotherapy and surveillance may be performed in this population, and more effective approaches should be studied.

\section{DATA AVAILABILITY STATEMENT}

The raw data supporting the conclusions of this article will be made available by the authors, without undue reservation.

\section{AUTHOR CONTRIBUTIONS}

YY and HX participated in the design of the study, performed the data management, statistical analysis and interpretation and 
were co-primary investigators. GC and YP were major contributors to the design of this study and revised the manuscript. All authors contributed to the article and approved the submitted version.

\section{REFERENCES}

1. Kapiteijn E, Marijnen CAM, Nagtegaal ID, Putter H, Steup WH, Wiggers T, et al. Preoperative Radiotherapy Combined With Total Mesorectal Excision for Resectable Rectal Cancer. New Engl J Med (2001) 345(9):638-46. doi: 10.1056/NEJMoa010580

2. Swedish Rectal Cancer T. Improved Survival With Preoperative Radiotherapy in Resectable Rectal Cancer. New Engl J Med (1997) 336(14):980-7. doi: 10.1056/NEJM199704033361402

3. Sauer R, Liersch T, Merkel S, Fietkau R, Hohenberger W, Hess C, et al. Preoperative Versus Postoperative Chemoradiotherapy for Locally Advanced Rectal Cancer: Results of the German CAO/ARO/AIO-94 Randomized Phase III Trial After a Median Follow-Up of 11 Years. J Clin Oncol (2012) 30 (16):1926-33. doi: 10.1200/JCO.2011.40.1836

4. Rodel C, Martus P, Papadoupolos T, Fuzesi L, Klimpfinger M, Fietkau R, et al. Prognostic Significance of Tumor Regression After Preoperative Chemoradiotherapy for Rectal Cancer. J Clin Oncol (2005) 23(34):8688-96. doi: 10.1200/JCO.2005.02.1329

5. Shia J, Guillem JG, Moore HG, Tickoo SK, Qin J, Ruo L, et al. Patterns of Morphologic Alteration in Residual Rectal Carcinoma Following Preoperative Chemoradiation and Their Association With Long-Term Outcome. Am J Surg Pathol (2004) 28(2):215-23. doi: 10.1097/00000478-200402000-00009

6. van Gijn W, Marijnen CAM, Nagtegaal ID, Kranenbarg EM-K, Putter H, Wiggers T, et al. Preoperative Radiotherapy Combined With Total Mesorectal Excision for Resectable Rectal Cancer: 12-Year Follow-Up of the Multicentre, Randomised Controlled TME Trial. Lancet Oncol (2011) 12(6):575-82. doi: 10.1016/S1470-2045(11)70097-3

7. Park IJ, You YN, Agarwal A, Skibber JM, Rodriguez-Bigas MA, Eng C, et al. Neoadjuvant Treatment Response As an Early Response Indicator for Patients With Rectal Cancer. J Clin Oncol (2012) 30(15):1770-6. doi: 10.1200/JCO.2011.39.7901

8. Collette L, Bosset J-F, den Dulk M, Nguyen F, Mineur L, Maingon P, et al. Patients With Curative Resection of cT3-4 Rectal Cancer After Preoperative Radiotherapy or Radiochemotherapy: Does Anybody Benefit From Adjuvant Fluorouracil-Based Chemotherapy? A Trial of the European Organisation for Research and Treatment of Cancer Radiation Oncology Group. J Clin Oncol (2007) 25(28):4379-86. doi: 10.1200/JCO.2007.11.9685

9. Govindarajan A, Reidy D, Weiser MR, Paty PB, Temple LK, Guillem JG, et al. Recurrence Rates and Prognostic Factors in ypN0 Rectal Cancer After Neoadjuvant Chemoradiation and Total Mesorectal Excision. Ann Surg Oncol (2011) 18(13):3666-72. doi: 10.1245/s10434-011-1788-y

10. Yu SKT, Tait D, Chau I, Brown G. MRI Predictive Factors for Tumor Response in Rectal Cancer Following Neoadjuvant Chemoradiation

\section{ACKNOWLEDGMENTS}

We thank Shanjun Huang from Department of General Surgery for data management and patient follow-up.

Therapy - Implications for Induction Chemotherapy? Int J Radiat Oncol Biol Phys (2013) 87(3):505-11. doi: 10.1016/j.ijrobp.2013.06.2052

11. Pang X, Gao Y, Yi H, Liu H, Liu S, Zheng J. Associations Between Clinical Characteristics and Tumor Response to Neoadjuvant Chemoradiotherapy in Rectal Cancer. Cancer Med (2021) 10(14):4832-43. doi: 10.1002/cam4.4051

12. Patel UB, Taylor F, Blomqvist L, George C, Evans H, Tekkis P, et al. Magnetic Resonance Imaging-Detected Tumor Response for Locally Advanced Rectal Cancer Predicts Survival Outcomes: MERCURY Experience. J Clin Oncol (2011) 29(28):3753-60. doi: 10.1200/JCO.2011.34.9068

13. Fokas E, Liersch T, Fietkau R, Hohenberger W, Beissbarth T, Hess C, et al. Tumor Regression Grading After Preoperative Chemoradiotherapy for Locally Advanced Rectal Carcinoma Revisited: Updated Results of the CAO/ARO/AIO-94 Trial. J Clin Oncol (2014) 32(15):1554-62. doi: 10.1200/ JCO.2013.54.3769

14. Lim S-B, Choi HS, Jeong S-Y, Kim DY, Jung KH, Hong YS, et al. Optimal Surgery Time After Preoperative Chemoradiotherapy for Locally Advanced Rectal Cancers. Ann Surg (2008) 248(2):243-51. doi: 10.1097/SLA. $0 \mathrm{~b} 013 \mathrm{e} 31817 \mathrm{fc} 2 \mathrm{a} 0$

15. Moore HG, Gittleman AE, Minsky BD, Wong D, Paty PB, Weiser M, et al. Rate of Pathologic Complete Response With Increased Interval Between Preoperative Combined Modality Therapy and Rectal Cancer Resection. Dis Colon Rectum (2004) 47(3):279-86. doi: 10.1007/s10350-003-0062-1

16. Brown T, Janjan NA, Feig BW, Cleary K, Dubrow R, Curley S, et al. Improved Overall Survival Among Responders to Preoperative Chemoradiation for Locally Advanced Rectal Cancer. Int J Radiat Oncol Biol Phys (1999) 45(3 SUPPL.):339-. doi: 10.1016/S0360-3016(99)90392-9

Conflict of Interest: The authors declare that the research was conducted in the absence of any commercial or financial relationships that could be construed as a potential conflict of interest.

Publisher's Note: All claims expressed in this article are solely those of the authors and do not necessarily represent those of their affiliated organizations, or those of the publisher, the editors and the reviewers. Any product that may be evaluated in this article, or claim that may be made by its manufacturer, is not guaranteed or endorsed by the publisher.

Copyright (c) 2021 Yang, Xu, Chen and Pan. This is an open-access article distributed under the terms of the Creative Commons Attribution License (CC BY). The use, distribution or reproduction in other forums is permitted, provided the original author(s) and the copyright owner(s) are credited and that the original publication in this journal is cited, in accordance with accepted academic practice. No use, distribution or reproduction is permitted which does not comply with these terms. 